\title{
Analysis of the Chemo-thermal Stresses around a Cylindrical Hole in a Saturated Porous Medium
}

\author{
Liang Chen ${ }^{1, \mathrm{a}}$, Qing Yao ${ }^{1, \mathrm{~b}}$, Luwu $\mathrm{He}^{1, \mathrm{c}^{*}}$ \\ ${ }^{1}$ School of Mechanical and Power Engineering, East China University of Science and Technology, \\ Shanghai 200237, China
}

agodloveliang@163.com, byaoqing@mail.ecust.edu.cn, 'Luwuhe@ecust.edu.cn

\begin{abstract}
Keywords: Saturated porous medium; Local thermal non-equilibrium; Laplace transform; Pore pressure; Chemo-thermal stress.
\end{abstract}

Abstract: In this paper, based on the chemo-thermo-poroelasticity model of saturated porous medium undergoing the local thermal non-equilibrium (LTNE) conditions, the pore pressure and chemo-thermal stresses around a cylindrical hole in an infinite fluid saturated porous medium subjected to sudden variations for temperature and solute mole fraction on the cylindrical hole boundary are investigated by employing Laplace transform technique and numerical method. The results show that the pore pressure and stresses are dominantly affected by the chemical effect rather than the thermal effect when the temperature and variation of the solute mole fraction change together, and the LTNE effects on radial stress (magnitude) become very pronounced when $S_{p}$ is small $(\approx 0.35)$ and $\mathrm{Bi}$ is moderate $(\approx 1.0)$. In addition, the pore pressure and stresses are same order of magnitude when considering the chemical effect or the chemo-thermal effect.

\section{Introduction}

Wellbore instability caused by the failure of rock formations represents a major economic challenge to the petroleum industry. During the drilling of a borehole, the rock matrix deforms under the drilling fluid pressure and tectonic stresses. Thermal stresses are also induced due to the temperature difference between the drilling fluid and the rock matrix. Moreover, the difference between the solute concentrations of chemical species in the drilling fluid and in the pore fluid causes solute diffusion in the rock which alters the stress state in the rock matrix. Hence, a complete understanding of rock failure and wellbore instability requires a multidisciplinary approach to take chemical, thermal and mechanical effects into account. Sherwood ${ }^{[1,2]}$ proposed a modification of the Boit poroelastic theory ${ }^{[3]}$ to include ionic transport effects on the pore pressure and stresses in shale rocks. The modified theory was used by Sherwood and Bailey ${ }^{[4]}$ to study shale swelling around a wellbore. Ghassemi and Diek ${ }^{[5]}$ presented a linear chemo-poroelastic model for shale formations. Ekbote and Abousleiman ${ }^{[6]}$ developed a poromechanics formulation for chemically active porous media and presented some solutions of stresses and pore pressure around a borehole. Temperature gradients in a porous medium saturated with a chemically active fluid not only induce thermal stresses but also influence the transport of solute and solvent. Ekbote and Abousleiman ${ }^{\text {[7] }}$ and Ghassemi and Diek ${ }^{[8]}$ modified the constitute relations in thermo-poroelasticity by considering the effects of solute concentration on the strains, temperature and variations of solute and solvent contents. Chemo-thermal stresses around a borehole were calculated in [7, 8].

The chemo-thermo-poroelasticity models of $[7,8]$ used the classical local thermal equilibrium (LTE) assumption, i.e., the solid and fluid reach thermal equilibrium instantaneously and the temperatures of the solid and fluid at a macroscopic point remain the same [9-11]. The LTE assumption remains adequate for many engineering and geophysical applications when the Sparrow number (or equivalently Nield number) ${ }^{[12,13]}$ is very large, which corresponds to large interstitial heat 
transfer coefficients between the solid and fluid phases and high pore surface area to pore volume ratios. For applications with small Sparrow numbers, local thermal non-equilibrium (LTNE) becomes significant, which promotes heat transfer between the solid and fluid and induces additional solute diffusion and pore pressure and chemo-thermal stresses in the porous medium.

In the present paper, we present a chemo-thermo-poroelasticity model for an isotropic porous medium saturated with a chemically active fluid. The model consists of LTNE heat transfer theory ${ }^{\text {[14] }}$ and uses a weight average temperature of the fluid and solid phases to describe the effects of LTNE in the constitutive relations. The model reduces to our LTNE thermo-poroelasticity model ${ }^{[15,16]}$ when the chemical effect is not present and the model of Ekbote and Abousleiman ${ }^{[7]}$ when LTE conditions prevail, respectively. Then, we obtain the pore pressure and chemo-thermal stress distributions around a cylindrical hole in an infinite porous medium using the Laplace transform method. Numerical examples are then presented to show the combined effects of solute transport and LTNE on the pore pressure and stress distributions around the hole.

\section{Governing equations}

Temperature field

Assuming isotropic porous elastic media without heat source, under the LTNE conditions, the solid and fluid phase temperatures are governed by the following heat conduction equations ${ }^{[14]}$

$$
\begin{aligned}
(1-\phi) \rho_{s} c_{s} \frac{\partial \theta_{s}}{\partial t}-(1-\phi) k_{T s} \Delta \theta_{s}+h_{\mathrm{int}}\left(\theta_{s}-\theta_{f}\right) & =0 \\
\phi \rho_{f} c_{f} \frac{\partial \theta_{f}}{\partial t}-\phi k_{T f} \Delta \theta_{f}+h_{\mathrm{int}}\left(\theta_{f}-\theta_{s}\right) & =0
\end{aligned}
$$

where $\theta_{s}$ and $\theta_{f}$ are the solid and fluid temperature variations respectively, $t$ is time, $\phi$ is the reference porosity, $k_{T f}$ and $k_{T s}$ is the thermal conductivities of the solid and fluid respectively, $\rho_{s}$ and $\rho_{f}$ are the densities for the solid and fluid respectively, $c_{s}$ and $c_{f}$ are the specific heat for the solid and fluid respectively, $h_{\text {int }}$ is the solid-fluid interface heat transfer coefficient, and $\Delta$ is the Laplacian operator. In addition, for the LTE conditions (i.e., temperature of the porous medium $\theta=\theta_{s} \equiv \theta_{f}$ ), the fluid-saturated porous medium temperature field can be simplified to the following form.

$$
\frac{\partial \theta}{\partial t}=\kappa \Delta \theta \quad(t>0)
$$

Here $\kappa$ is the effective thermal diffusivity of fluid saturated porous medium ${ }^{[15,16]}$.

Taking into account the case of the structure and the loads, the analysis of the chemo-thermal stresses around a cylindrical hole in an infinite saturated porous medium can be simplified to a axisymmetric plane problem, shown as Fig.1. $a$ is hole radius, $r$ is radial coordinate in polar coordinates. The initial and boundary conditions for the temperatures can thus be formulated as follows:

$$
\begin{gathered}
\left.\theta_{s}(r, t)\right|_{t=0}=\left.\theta_{f}(r, t)\right|_{t=0}=0, \quad a \leq r<\infty \\
-\left.k_{T s} \frac{\partial \theta_{s}(r, t)}{\partial r}\right|_{r=a}=\left.h_{T s}\left(\theta_{f}-\theta_{s}\right)\right|_{r=a},\left.\quad \theta_{f}(r, t)\right|_{r=a}=\theta_{a} H(t), \quad t>0 \\
\left.\theta_{s}(r, t)\right|_{r \rightarrow \infty}=0,\left.\quad \theta_{f}(r, t)\right|_{r \rightarrow \infty}=0, \quad t>0
\end{gathered}
$$

where, $\theta_{a}$ is a constant (the temperature of the drilling fluid) and $H(t)$ is the Heaviside step function, 
$h_{\mathrm{Ts}}$ is the heat transfer coefficient. When the Biot number $B i\left(=1 / b_{4 s}=a h_{T s} / k_{T s}\right)$ is very large, equation (4) will be degraded to the first boundary condition, that is the temperature of solid phase and flow phase at the boundary are given and same.

In the classical local thermal equilibrium heat transfer theory (LTE conditions, $\theta=\theta_{s} \equiv \theta_{f}$ ), the corresponding initial and boundary conditions of the heat conduction problem are

$$
\begin{gathered}
\left.\theta(r, t)\right|_{t=0}=0, \quad a \leq r<\infty \\
-\left.k_{T m} \frac{\partial \theta(r, t)}{\partial r}\right|_{r=a}=\left.h_{T m}\left(\theta_{a} H(t)-\theta\right)\right|_{r=a},\left.\quad \theta(r, t)\right|_{r \rightarrow \infty}=0, \quad t>0
\end{gathered}
$$

where $k_{T m}$ is the effective thermal conductivity of saturated porous media, $h_{T m}$ is the heat transfer coefficient between porous media and the drilling fluid.

For the two initial-boundary value problems, (1), (3) and (4); (2), (5) and (6), the analytical expression of $\theta_{s}, \theta_{f}$ and $\theta$ in a Laplace transform domain can be obtained by Laplace transform technique.

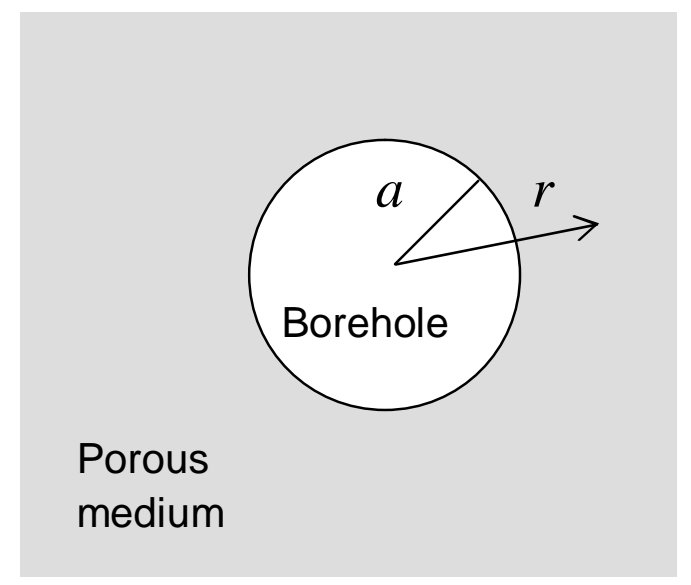

Fig. 1 A cylindrical hole in an infinite fluid saturated porous medium

The chemo-thermal stresses of poroelastic media

Based on the weighted average temperature concepts ${ }^{[15,16]}$ and the works of Ekbote and Abousleiman $^{[6,7]}$, for the saturated porous medium, in which the pore fluid/solution consists of two components (solvent and solute), the governing equations for the displacement, pore pressure and solute molar fraction variation under the LTNE conditions can be written as follows ${ }^{[17]}$ :

$$
\begin{gathered}
G \Delta\left(u_{i}\right)+\frac{G}{1-2 v} u_{k, k i}-\alpha p_{, i}-K \alpha_{s} \theta_{\text {ave }, i}+\gamma^{s} m_{, i}^{s}=0 \\
\frac{1}{M} \frac{\partial p}{\partial t}+C_{s} \frac{\partial m^{s}}{\partial t}=B_{p s} \Delta p+B_{m s} \Delta m^{s}+B_{T s} \Delta \theta_{a v e}-\alpha \frac{\partial\left(\varepsilon_{k k}\right)}{\partial t}+\beta^{T s} \frac{\partial \theta_{a v e}}{\partial t} \\
\frac{1}{M} \frac{\partial p}{\partial t}+C_{f} \frac{\partial m^{s}}{\partial t}=B_{p f} \Delta p+B_{m f} \Delta m^{s}+B_{T f} \Delta \theta_{a v e}-\alpha \frac{\partial\left(\varepsilon_{k k}\right)}{\partial t}+\beta^{T f} \frac{\partial \theta_{a v e}}{\partial t}
\end{gathered}
$$

where $u_{i}$ is the displacement of solid-phase, $p$ is pore pressure of fluid, $m^{s}$ is the variation of solute molar fraction, $\varepsilon_{k k}=u_{k, k}, \theta_{\text {ave }}=(1-\phi) \theta_{s}+\phi \theta_{f}$.

For an infinite medium under the irrotational displacement field condition, namely $u_{i, j}=u_{j, i}$, there will be $\Delta u_{i}=\varepsilon_{k k, i}$, and equations (7)-(9) can be simplified as follows: 


$$
\begin{gathered}
\varepsilon_{k k}=\frac{\alpha}{(\lambda+2 G)} p+\frac{K \alpha_{s}}{(\lambda+2 G)} \theta_{\text {ave }}-\frac{\gamma^{s}}{(\lambda+2 G)} m^{s} \\
\frac{\partial p}{\partial t}=a_{11} \Delta p+a_{12} \Delta m^{s}+a_{13} \Delta \theta_{\text {ave }}+a_{14} \frac{\partial \theta_{\text {ave }}}{\partial t} \\
\frac{\partial m^{s}}{\partial t}=a_{21} \Delta p+a_{22} \Delta m^{s}+a_{23} \Delta \theta_{\text {ave }}+a_{24} \frac{\partial \theta_{\text {ave }}}{\partial t}
\end{gathered}
$$

here the definition and physical meaning of the coefficients in equations (7)-(12) can refer to the Reference [17]. The above equations (10)-(12) present that the pore pressure, variation of solute molar fraction and the displacements become uncoupled when the displacement field is irrotational in an infinite medium.

For the infinite fluid saturated isotropic porous medium with a cylindrical hole, shown as Fig.1, assuming that the initial state is the natural state, the pore pressure and the solute molar fraction variation respectively suffer to a sudden increment at the edge of $\operatorname{hole}(r=a)$, and the solid-phase is free, at this time, the initial and boundary conditions can be expressed as follows:

$$
\begin{array}{cc}
\left.\sigma_{r}(r, t)\right|_{t=0}=\left.u(r, t)\right|_{t=0}=\left.p(r, t)\right|_{t=0}=\left.m^{s}(r, t)\right|_{t=0}=0, & a \leq r<\infty \\
\left.p(r, t)\right|_{r=a}=p_{a} H(t),\left.\quad p(r, t)\right|_{r \rightarrow \infty} \rightarrow 0, & t>0 \\
\left.m^{s}(r, t)\right|_{r=a}=m_{a}^{s} H(t),\left.\quad m^{s}(r, t)\right|_{r \rightarrow \infty} \rightarrow 0, & t>0 \\
\left.\sigma_{r}(r, t)\right|_{r=a}=-p_{a} H(t),\left.\quad \sigma_{r}(r, t)\right|_{r \rightarrow \infty} \rightarrow 0, & t>0
\end{array}
$$

where $p_{a}$ and $m_{a}^{s}$ are the sudden increments (constant) of pore pressure and molar fraction of solute respectively, $u$ is the radial displacement of the solid phase, $\sigma_{r}(r, t)$ is the radial stress of the solid phase.

For the axisymmetric plane problem (plane strain problem), the solid phase displacement is irrotational field, therefore, the pore pressure $p$ and the solute molar fraction $m^{s}$ can be firstly solved by the equations (11) and (12). Combined with the initial and boundary conditions (13)-(15) and the expressions of $\theta_{s}$ and $\theta_{f}$ in the Laplace transform domain, the expressions of $p$ and $m^{s}$ in Laplace transform domain can be obtained by Laplace transform from equations (11) and (12). After obtaining the temperature filed of solid-phase and fluid-phase $\theta_{s}$ and $\theta_{f}\left(\theta_{\text {ave }}=(1-\phi) \theta_{s}+\phi \theta_{f}\right)$, and the pore pressure $p$ and the variation of solute molar fraction $m^{s}$, the radial displacement $u$ and the radial stress $\sigma_{r}(r, t)$ and the tangential normal stress $\sigma_{\theta}(r, t)$ of solid phase can be obtained by the equation of (10) with initial and boundary conditions (13) and (16).

\section{Numerical results}

Since the expressions of temperature field, pore pressure and solute molar fraction in Laplace transform domain are very complicated, the numerical method ${ }^{[18]}$ for the inverse Laplace transform is suitable. In this paper, the clay material as solid phase is considered for numerical computation and analysis, and the specific heat - elastic material parameters (clay) are taken from the References $[15,16]$, i.e., $: \phi=0.2, G=6.8 \mathrm{GPa}, \nu=0.18, K=8.4 \mathrm{G} \mathrm{Pa}, \mu=1.0 \times 10^{-3} \mathrm{~Pa} \cdot \mathrm{s}, k=2.0 \times 10^{-16} \mathrm{~m}^{2}, \alpha=0.74, \rho_{s}$ $=2600 \mathrm{~kg} / \mathrm{m}^{3}, \quad \rho_{f}=1000 \mathrm{~kg} / \mathrm{m}^{3}, \quad c_{s}=920 \mathrm{~J} /\left(\mathrm{kg}^{0} \mathrm{C}\right), \quad c_{f}=4200 \mathrm{~J} /\left(\mathrm{kg}^{0} \mathrm{C}\right), \quad k_{T s}=2.4 \mathrm{~W} /\left(\mathrm{m}^{0} \mathrm{C}\right), \quad k_{T f}$ $=0.6 \mathrm{~W} /\left(\mathrm{m}^{0} \mathrm{C}\right), \alpha_{\mathrm{s}}=3.3 \times 10^{-5} /{ }^{0} \mathrm{C}, \alpha^{f}=\alpha^{s}=3.0 \times 10^{-4} /{ }^{0} \mathrm{C}$. The chemical parameters refer to the References 
[5-8]: $\gamma^{s}=100 \mathrm{MPa}, \bar{m}^{s}=0.15, \bar{T}=300 \mathrm{~K}, V_{\mathrm{s}}=3.36 \times 10^{-5} \mathrm{~m}^{3} / \mathrm{mol}$ (Nacl solute), $V_{f}=1.8 \times 10^{-5} \mathrm{~m}^{3} / \mathrm{mol}$ (hydrosolvent), $\chi=0.9, R=8.314 \mathrm{~J} /{ }^{0} \mathrm{C}-\mathrm{mol}, D^{c}=2.0 \times 10^{-9} \mathrm{~m}^{2} / \mathrm{s}, D^{T s}=D^{T f}=1.5 \times 10^{-9} \mathrm{~m}^{2} / \mathrm{s}, a=0.1 \mathrm{~m}$. The relevant material parameters of isotropic saturated porous media are set as constant, and calculated as follows: $c^{\prime}=4.641 \times 10^{5} \mathrm{~Pa} /{ }^{0} \mathrm{C}, \quad \kappa=5.427 \times 10^{-7} \mathrm{~m}^{2} / \mathrm{s}, \quad b_{3}=0.354$ 。

The following mainly discusses the influence of pore pressure and chemo-thermal stress, and LTNE effect and chemical effect. Let $b_{5}=k_{T m} /\left(a h_{T m}\right)=b_{4 s}$, and take $p_{a}=0$ and $\tilde{h}=a^{2} h_{\text {int }} /(1-\phi) b_{3} k_{T s}=1.0$ (the Sparrow number $S_{p} \cong \tilde{h} b_{3}=0.354$ ), $m_{a}^{s}=-1.8 \times 10^{-2}$ ( the concentration of solutes $\mathrm{NaCl}$ reduces $1 \mathrm{~mol} / \mathrm{kg}$ ), $\theta_{L}=50{ }^{0} \mathrm{C}$. If the temperature and solute molar fraction change together (chemo-thermal effect), take $\theta_{a}=\theta_{L}$. When only consider the molar fraction of solute changes (chemical effect), take $\theta_{a}=0$.
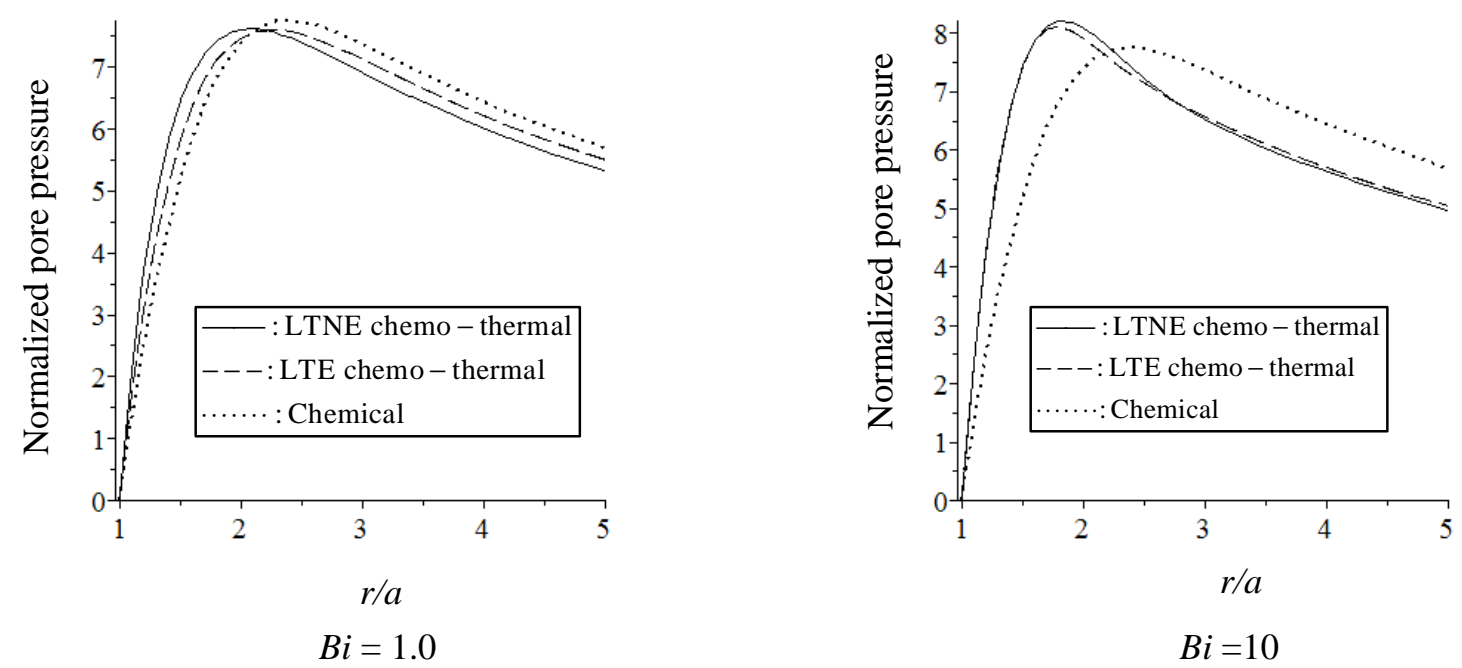

Fig. 2 Normalized pore pressure distributions along the radial direction ( $B i=1.0$ and $B i=10)$

Figure. 2 show the radial distribution of normalized pore pressure $p /\left(c, \theta_{a} \times 10^{-2}\right)$ at a nondimensional time $\tilde{t}=\kappa t / a^{2}=0.1$. The Biot numbers $B i$ are 1.0 and 10 in Fig. 2, respectively. Pure chemical, LTNE chemo-thermal, and LTE chemo-thermal effects are considered. It can be seen that the pore pressure first increases with $r / a$, reaches a peak value, and then decreases with further increasing radial distance from the hole boundary. The pore pressure is dominated by the chemical effect as the chemo-thermal pore pressures deviate slightly from that due to pure chemical effect. The peak normalized pore pressure under only chemical effect is now 7.75 while the peak normalized values of the chemo-thermal pressure are 7.62 and 7.59 under LTNE and LTE conditions, respectively, when the Biot number is $B i=1.0$. For $B i=10.0$, LTNE and LTE chemo-thermal pore pressures increase to 8.21 and 8.10 , respectively, which are $6 \%$ and $4.5 \%$ higher than the peak pore pressure due to chemical effect only.

Figure. 3 show the normalized radial stress $\sigma_{r} /\left(c^{\prime} \theta_{a} \times 10^{-2}\right)$ distribution along the radial direction at a nondimensional time $\tilde{t}=\kappa t / a^{2}=0.1$ for $B i=1.0$ and $B i=10$, respectively. It can be observed that the radial stresses are compressive and their magnitudes first increase with $r / a$, reach a peak value, and then decrease with further increasing distance. Among the three cases considered (pure chemical, LTNE chemo-thermal and LTE chemo-thermal), the chemical stress has the minimum magnitude and the LTNE chemo-thermal stress has the highest magnitude, which indicates that the LTNE effect is significant. The peak magnitude of the chemical stress is 2.2. The LTNE and LTE chemo-thermal stresses have peaks of 4.2 and 3.0, respectively for, $B i=1.0$. The peak magnitudes of the stresses increase with an increase in Biot number. The difference between the LTNE and LTE stresses becomes smaller for the larger Biot number case. 


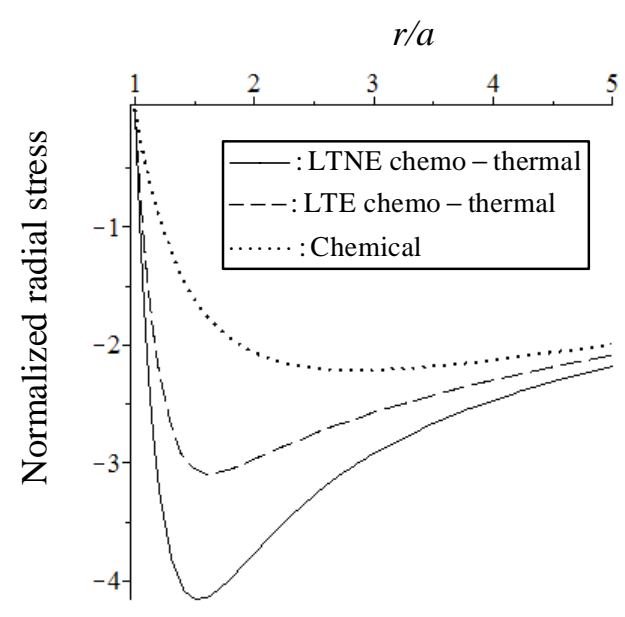

$B i=1.0$

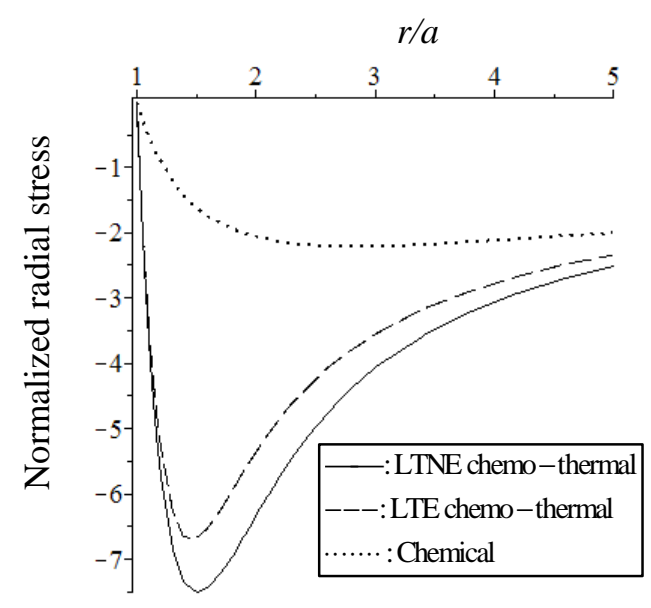

$B i=10$

Fig. 3 Normalized radial stress distributions along the radial direction ( $B i=1.0$ and $B i=10$ )

Figure.4 show the normalized tangential stress $\sigma_{\theta} /\left(c, \theta_{a} \times 10^{-2}\right)$ distribution along the radial direction at a nondimensional time $t^{\sim}=\kappa t / a^{2}=0.1$. The Biot numbers are $B i=1.0$ and $B i=10$, respectively. First, the tangential stresses are mostly compressive and their magnitudes decrease with increasing distance from the hole boundary. Among the three cases considered, again the chemical stress has the minimum magnitude and the LTNE chemo-thermal stress has the highest magnitude. Moreover, the chemical stress is significantly lower than the LTNE chemo-thermal stress. The peak magnitude of chemical stress is 6.0 whereas the LTNE and LTE chemo-thermal stresses have peaks of 27 and 18, respectively, for $B i=1.0$. The peak magnitudes of the stresses increase with an increase in Biot number. Again, the difference between the LTNE and LTE stresses become smaller for the larger Biot number. The results in Figs. 2-4 indicate that chemical effect play a major role in pore pressure increase around the hole whereas LTNE thermal effects dominate in radial and tangential stress variations.
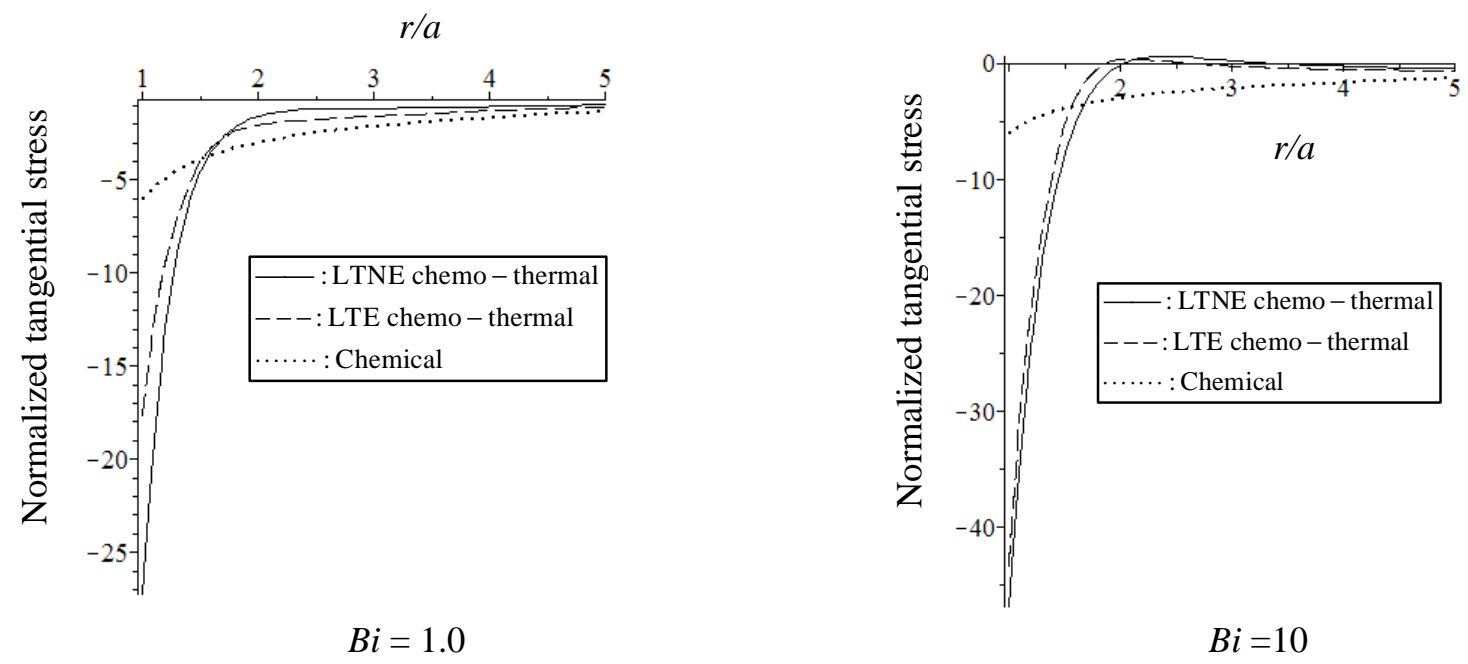

Fig. 4 Normalized tangential stress distributions along the radial direction ( $B i=1.0$ and $B i=10)$

\section{Conclusion}

In this paper, we present a local thermal non-equilibrium (LTNE) chemo-thermo-poroelasticity model for fluid saturated porous media. The combined effects of LTNE and solute transport on the pore pressure and stresses are examined using a cylindrical hole in an infinite porous medium with the 
hole boundary subjected to the action of a chemically active fluid. Moreover, LTNE also influences solute diffusion in a temperature gradient, which indirectly induces additional pore pressure and stresses. It is shown that under the LTNE conditions, solute transport has a more pronounced effect on the chemo-thermally induced pore pressure and stresses. The LTNE chemo-thermal pore pressure and the magnitudes of the chemo-thermal stresses may become significantly higher than those under classical local thermal equilibrium conditions.

\section{References}

[1] Sherwood J D. A model for the flow of water and ions into swelling shale[J]. Langmuir, 1994, 10: 2480-2486.

[2] Sherwood J D. Ionic transport in swelling shale[J]. Adv. Colloid Interface Sci., 1995, 61:51-64.

[3] Biot M A. General Three-dimensional Theory of Poroelasticity[J]. J. Appl. Phys., 1941, 12: $155-164$.

[4] Sherwood J D, Bailey L. Swelling shale around a cylindrical wellbore[J]. Proc. R. Soc. London, Ser. A, 1994, 444:161-184.

[5] Ghassemi A, Diek A. Linear chemo-poroelasticity for swelling shales: theory and application[J]. Journal of Petroleum Science and Engineering, 2003, 38:199-212.

[6] Ekbote S, Abousleiman Y. Porochemoelastic solution for an inclined borehole in a transversely isotropic formation[J]. ASCE Journal of Engineering Mechanics, 2006, 132:754-763.

[7] Ekbote S, Abousleiman Y. Porochemothermoelastic solution for an inclined borehole in a transversely isotropic formation[J]. ASCE Journal of Engineering Mechanics, 2005, 131: 522-533.

[8] Ghassemi A, Diek A. Influence of coupled chemo-poro-thermoelastic processes on pore pressure and stress distributions around a wellbore in a swelling shale[J]. Journal of Petroleum Science and Engineering, 2009, 67:57-64.

[9] McTigue D F. Thermal response of fluid-saturated porous rock[J]. Journal of Geophysical Research, 1986, 91 (B9):9533-9542.

[10] Kurashige M. A thermoelastic theory of fluid-filled porous materials[J]. International Journal of Solids and Structures, 1989, 25:1039-1052.

[11] Y. Abousleiman Y, Ekbote S. Solutions for the inclined borehole in a porothermoelastic transversely isotropic medium[J]. ASME Journal Applied Mechanics, 2005, 72:102-114.

[12] Minkowycz W J, Haji-Sheikh A, Vafai K. On the departure from local thermal equilibrium in porous media due to a rapidly changing heat source: the Sparrow number [J]. International Journal of Heat and Mass Transfer, 2009, 42: 3373-3385.

[13] Nield D A. Effects of local thermal nonequilibrium in steady convection processes in saturated porous media: Forced convection in a channel[J]. J. Porous Media, 1998, 1:181-186.

[14] Nield D A, Bejan A. Convection in Porous Media[M]. $3^{\text {rd }}$ edition, Berlin: Springer, 2006.

[15] He L W, Jin Z H. A local thermal nonequilibrium poroelastic theory for fluid saturated porous media [J]. Journal of Thermal Stresses, 2010, 33:799-813.

[16] He L W, Jin Z H, Zhang Y. Convective cooling/heating induced thermal stresses in a fluid saturated porous medium undergoing local thermal non-equilibrium [J]. International Journal of Solids and Structures, 2012, 49: 748-758.

[17] Dai Q C. Chemo-thermal stresses analysis of saturated poroelastic media with a cylindrical hole or spherical cavity [D]. East China University of Science and Technology, Shanghai, 2014.

[18] Durbin F. Numerical inversion of Laplace transformation: an efficient improvement to Durbin and Abate's method [J]. The Computer Journal, 1974, 17(4): 371-376. 